DR. SARAH COPE (Orcid ID : 0000-0002-1672-9459)

Article type : Original Article

\title{
Enhancing the communication of functional neurological disorder diagnosis: A multi-disciplinary education session
}

Sarah R. Cope ${ }^{a}$, Jared G. Smith ${ }^{b}$, Mark J. Edwards ${ }^{c, d}$, Kate Holtc, and Niruj Agrawala,c

a Department of Neuropsychiatry, South West London and St. George's Mental Health NHS Trust, London, UK

b Population Health Research Institute, St George's, University of London, London, UK

c Department of Neurology, St George's University Hospitals NHS Foundation Trust, London, UK

d Neurosciences Research Centre, Molecular and Clinical Sciences Research Institute, St George's, University of London, London, UK

Corresponding author:

Dr Sarah R. Cope

Department of Neuropsychiatry

St. George's Hospital

Blackshaw Road

London

SW17 0QT

Tel: 02087253786

Fax: 02035134460

Email: sarah.cope@swlstg.nhs.uk

This article has been accepted for publication and undergone full peer review but has not been through the copyediting, typesetting, pagination and proofreading process, which may lead to differences between this version and the Version of Record. Please cite this article as doi: 10.1111/ENE.14525

This article is protected by copyright. All rights reserved 
Word count: 4358 (entire manuscript); 3247 (Introduction, Method, Results, Discussion only)

Key words: functional neurological disorder; conversion disorder; psychogenic; education; psychoeducation; communication

Disclosure of Conflict of Interest: None 


\section{Abstract}

Background: Functional neurological disorder (FND) is a common diagnosis within Neurology. Effective communication of the diagnosis is known to be an important part of treatment and can result in reduction or cessation of symptoms, as well as decreased healthcare utilisation. A single group education session, facilitated by professionals commonly involved in the care of patients with FND, was developed to further enhance patients' and relatives' understanding and acceptance of diagnosis.

Methods: Patients and relatives attending a single education session were asked to complete self-report ratings of understanding of diagnosis, acceptance of diagnosis, belief in treatability, and hopefulness regarding recovery, at the beginning and end of the session. Satisfaction data was also collected.

Results: Data was obtained from 193 patients and 153 relatives. Patients had experienced a median duration of symptoms of 4 years, and more than $80 \%$ of patients reported more than one functional neurological symptom. There were significant increases in terms of understanding, acceptance, belief in treatability and hopefulness for patients and relatives. Effect sizes ranged from large for improved understanding of FND to small-to-medium for increased agreement with FND diagnosis. High levels of satisfaction were also reported.

Conclusions: A multi-disciplinary single group education session is an effective and acceptable method of increasing understanding and acceptance of an FND diagnosis, even for patients with a long-duration of symptoms and high symptom burden. It could help improve readiness for further treatment.

\section{Introduction}

Functional neurological disorder (FND) is a common presentation in neurology clinics [1], which is associated with significant distress and disability [2]. FND describes the presence of genuine neurological symptoms (e.g. sensory loss, weakness, tremor, 
seizure-like episodes) that are present despite the apparent normal functioning of basic or low-level aspects of the motor and sensory system. Symptoms therefore represent a loss of access or control over the body: a disconnection between conscious perception/will and the body. Common presentations within FND are functional seizures (also know as psychogenic non-epileptic seizures, functional non-epileptic attacks, and dissociative seizures) and functional motor symptoms (e.g. weakness, tremor, jerks).

An established part of treatment is effective communication of the diagnosis, and different groups have proposed communication protocols for the diagnosis of functional seizures [3-6], and discussed how to effectively explain the diagnosis of FND in neurology appointments $[7,8]$. Effective communication of the diagnosis can result in a reduction or resolution of symptoms. For example, Hall-Patch and colleagues reported that in a group of patients experiencing functional seizures, who were told their diagnosis according to the communication strategy they designed, $6 \%$ were seizure-free 2 weeks following diagnosis and this rose to $14 \% 11$ weeks after diagnosis [6]. Clear communication of diagnosis is thought to not only be therapeutic, but has also been found to reduce healthcare service use $[9,10]$.

Education for FND, consisting of more sessions, has reported some positive outcomes in both an individual format (4-sessions) [11], and group format (4-session CBT-informed psycho-education [12], 3-session psychoeducation [13], and 3-session CBT-informed psychoeducation [14]). With the exception of one [12], all these interventions were for patients with functional seizures only, and provided more than education - they also introduced ideas regarding management and coping. Chen and colleagues intervention was the only intervention that encouraged relatives to attend too [13]. It has been found that relatives of those with FND tend to hold more negative illness perceptions, compared to the patients with FND; therefore, including relatives in an intervention could help to reduce any negative views they may hold, which may in turn provide a more hopeful environment for patients [15].

FND can be a difficult condition for people (including health professionals) to understand, and therefore explanations may need to be given more than once by different healthcare professionals (e.g. neurologists, neuropsychiatrists, clinical psychologists, 
physiotherapists). Multiple explanations can lead to mixed messages as different professionals can use different language. In order to enhance understanding about FND diagnosis, we developed a multi-disciplinary education session for patients and their relatives. We hoped that the group format would also enable patients to meet others with FND, helping them to see that the condition is not rare, and reduce stigma and distress associated with the diagnosis.

\section{Method}

\section{Referrals and recruitment}

Referrals were made by regional neurologists and neuropsychiatrists following a clinical diagnosis of FND. Inclusion criteria were a primary diagnosis of: functional seizures, functional movement disorder, functional cognitive symptoms, functional weakness or sensory symptoms, or functional blindness. Referrers were asked to seek verbal consent from patients for their details to be passed on to the session organisers, and were also asked to obtain consent to be contacted via email. Patients were emailed or posted an invitation to attend the next session, which asked them to confirm their attendance, as well as specify how many relatives/friends/carers they would be bringing (a maximum of two). The term relative is used to refer to any relative or friend or carer the patient chose to bring.

At the session, patients and their relatives were asked to complete questionnaires at the beginning and end of the session. Informed consent was obtained for using their anonymised responses to evaluate the session and to publish the results in scientific literature. It was registered as a service evaluation with the relevant NHS Trust, therefore ethical approval was not needed. 


\section{Self-Report Measures}

Demographic information

Patients and relatives were asked to indicate their age, gender, ethnic background, and highest education level.

\section{Self-reported symptoms}

Patients were asked to circle the FND symptoms they experienced from the following list: Fits or seizures, Muscle spasms, Tingling/Numbness, Limb weakness, Difficulty walking, Memory problems, and Other (Please specify). They were also asked to write down how many years they had experienced the symptoms.

Relationship to patient

Relatives were asked to indicate their relationship to the patient from the following options: Parent, Partner, Sibling, Other relative, Friend, Carer, and Other (please specify).

Views on diagnosis of FND

Patients and their relatives were asked to rate, on numerical rating scales ranging from 0100, how much they: understood the FND diagnosis; agreed with the diagnosis; were hopeful regarding recovery; and believed FND is treatable. Each scale had descriptive anchor points at each end, which were respectively: "I have no understanding" and "I understand completely"; "I totally disagree" and "I completely agree"; "I am not hopeful at all" and "I am extremely hopeful"; "I do not believe it is treatable" and "I completely believe it is treatable". This was completed at the beginning and the end of the session. 
EQ-5D-5L (descriptive system)

This measure was completed by patients at the beginning of the session. The EQ-5D-5L includes a descriptive system and a visual analogue scale indicating current health. Only the descriptive system was administered. This measures 5 dimensions: mobility, selfcare, usual activities, pain/discomfort and anxiety/depression; and has 5 levels of responses for each dimension ( $1=$ no problems, $2=$ =slight problems, $3=$ moderate problems, $4=$ severe problems, and $5=$ extreme problems) [16]. Overall EQ-5D-5L health state valuation scores were calculated using utilities published for England [17].

\section{Feedback form}

At the end of the session, patients and relatives were asked to fill in a feedback form.

They were asked to rate on 6-point scales: "satisfaction" (how satisfied they were with the session; scale responses ranged from 'Not at all satisfied' to 'Very satisfied'); "helpfulness" (whether attendance of the session will help them manage their symptoms/support their friend or family member; scale responses ranged from 'Not at all' to 'Very much'); and "recommend" (how likely they would be to recommend the session; scale responses ranged from 'Unlikely' to 'Very likely').

\section{Group Education Session Format}

The session was held in a lecture theatre with PowerPoint presentation and videos facilitated by a Consultant Neurologist (MJE), Consultant Neuropsychiatrist (NA), Clinical Psychologist (SC), Neuro-physiotherapist (KH), and a patient who had been through treatment. The session lasted 1 hour and 45 minutes. The first part was led by the neurologist, who covered assessment and diagnosis of functional neurological symptoms, the main types of FND presentations, and the various labels for FND. The 
second part was led by the neuropsychiatrist who spoke about aetiological aspects including common predisposing, precipitating, and perpetuating factors; and gave an overview of what to expect from neuropsychiatric assessment and treatment. The clinical psychologist introduced what psychological treatment may look like, using cognitivebehavioural therapy as an example; and discussed two fictional case examples. Following this, the neuro-physiotherapist discussed how specialist physiotherapy can help to improve physical function and reduce disability. An ex-patient then discussed their experiences of receiving a FND diagnosis, and their experiences of treatment. The audience were directed to relevant self-help resources including websites containing further information regarding FND, and patient-led organisations. Finally, there was a question and answer session, where the audience asked the facilitators questions; and patients/relatives were given the outcome measures to complete. This part lasted around 30 minutes. Patients and their relatives were given a handout with copies of the slides presented.

\section{Statistical Analyses}

Means $(M)$ and standard deviations (SD) were calculated for FND session questionnaire scale items and health function measures at pre- and/or post-intervention, and frequencies and percentages calculated for sociodemographic variables and (presession) indicators of clinical function. Sociodemographic characteristics of patients with and without relative(s) were compared using Chi-square tests, while comparisons of participants completing and not completing outcome measures according to sociodemographic and clinical variables were administered using Chi-square tests and independent groups $t$-tests/one-way analysis of variance (ANOVA), with a criterion for statistical significance set at $P<0.05$. In all comparisons involving sociodemographic characteristics, age was collapsed into three broad classifications (18-35 years, 36-55 years, 56+ years) and highest education level was dichotomised (degree versus school only).

To evaluate whether FND understanding, agreement with diagnosis, hopefulness of recovery and belief in treatability improved in a significant manner after the session for patients and their relatives, pairwise comparisons (paired sample t-tests) were 
administered and effect sizes (Cohen's d; [18]; calculated as a difference between a pretreatment score and mid-/post-treatment score divided by the pre-treatment SD) obtained. Where skewness and kurtosis estimates indicated that continuous variables were not normally distributed (acceptable range between -1 and +1 and -1.5 and +1.5 , respectively;[19]), bias corrected and accelerated (Bca) bootstrapping using 2000 replications [20] was employed to calculate $95 \%$ confidence intervals of mean difference and associated $P$ values, and effect sizes were calculated with values transformed to better approximate a normal distribution using Box-Cox methods [21]. To minimise the possibility of false-positive errors related to multiple outcome testing across questionnaire items, the false discovery rate (FDR) approach was applied to within-group comparisons, with control set to $5 \%$ [22].

Residualised change scores (RCS) for session questionnaire scale items were computed and comparisons of RCS and post-session measures (including satisfaction scores) between patients and relatives using (bootstrapped) independent group $t$-tests, with a criterion for statistical significance set at $P<0.05$. Exploratory associations of RCS with sociodemographic factors (patients and relatives) and presence of clinical symptoms and health function (patients) were examined using Pearson correlation coefficients (or Spearman's rho depending on distributional properties) and independent group $t$ tests/one-way ANOVA (for categorical variables). Statistical analyses were performed using SPSS statistical software (SPSS Release 24.0, IBM).

\section{Results}

\section{Attendance}

Across 11 sessions, 193 patients and 153 relatives attended and provided data. The mean number of patients and relatives per session was 17.7 (range 15-23) and 13.9 (range 11-18), respectively. Two patients attended two sessions during the period under consideration; only data relating to their second visit was considered in analyses. Just under a third of patients $(62 ; 32.1 \%)$ attended without an accompanying relative; most 
(115; 59.6\%) were accompanied by a single relative while a small number $(16 ; 8.3 \%)$ attended the session with two relatives. Four relatives attended without the invited patient, while another relative did not have information linked with a corresponding patient (this data was included in analyses). Relatives were predominantly a mix of patients' partners $(57 ; 38.0 \%)$ and parents $(52 ; 34.7 \%)$, with small numbers of siblings $(10 ; 6.7 \%)$, other relatives $(13 ; 8.7 \%)$, friends $(6 ; 4.0 \%)$, carers $(3 ; 2.0 \%)$ and others $(9$; $6.0 \%)$.

\section{Demographics and Clinical Profile}

The sociodemographic characteristics of participants is shown in Table 1. The majority of patients were female $(78.8 \%)$, with a wide age range. Relatives were evenly split between males and females and tended to be older than the patients they were accompanying. Most patients and relatives identified as white/British. A little more than a third of relatives were educated to a degree level; this rate increased to $40 \%$ in patients.

There was a trend indicating proportionally less male patients attended with a relative compared with female patients $(23 ; 56.1 \%$ versus $108 ; 71.1 \% ; \chi 2=8.03, P=0.069)$, while a significantly smaller proportion of patients who attended with a relative were educated to degree level than those who attended without a relative (38; $32.8 \%$ versus $31 ; 55.4 \% ; x 2=8.03, P=0.005)$.

\section{[Insert Table 1 about here]}

Table 2 shows the clinical data of participants. Patients had experienced symptoms for a median duration of 4 years (range 0.1-40 years). Patients reported experiencing a variety of symptoms associated with their condition, with more than $80 \%$ reporting more than one symptom and slightly less than half reporting 4 or more (mean number $=3.34, \mathrm{SD}=$ 1.73). Between $40 \%$ and $55 \%$ of patients reported experiencing each listed symptom, 
with spasms, weakness, memory problems and fits endorsed frequently. Symptom combinations varied considerably across patients, although fits or seizures were more often reported in isolation $(15 / 83 ; 18.1 \%)$ than other symptoms $(0 \%-8.6 \%$ across different symptoms).

\author{
[Insert Table 2 about here]
}

Quality of life was impaired in the FND sample (Table 2); patients' mean EQ-5D-5L health state valuation score (0.55) was markedly less than (EQ-5D-3L) norms observed in age-matched healthy UK populations (which across ten-year age cohorts from 25-75 years range from 0.93 to 0.78 ;[23]). Problems concerning pain/discomfort $(75.6 \%)$, mood disturbances (80.3\%) and carrying out usual activities (83.4\%) were domains most affected, with considerably higher rates than self-reported EQ-5D-5L problems observed in a large sample of the general population in England (53.6\%, 31.4\% and $30.2 \%$, respectively [24].

\title{
Session outcomes and satisfaction
}

Pre- and post-FND Education Session data was available for 163 (84.5\%) FND patients and $115(75.7 \%)$ relatives. A higher proportion of male patients $(11 ; 26.8 \%$ versus female, $19 ; 12.5 \% ; X 2=5.05, P=0.025)$ and patients who reported experiencing fits or seizures $(17 ; 20.5 \% \%$ versus no fits or seizures, $10: 9.3 \% ; \chi 2=4.76, P=0.029)$ did not complete outcome data. But no other sociodemographic or clinical variable was significantly associated with measure completion in patients and no (sociodemographic) variables were related to relatives' measure completion. 
Significant increases between pre- and post-session ratings of understanding of diagnosis, belief in treatability, hopefulness regarding recovery, and agreement with diagnosis were observed for both patients and relatives (Table 3), all of which survived correction for multiple comparisons. Effect sizes ranged from large for improved understanding of FND to small-to-medium for increased agreement with FND diagnosis. Across all response domains, both the degree of change (after controlling for relevant baseline score) from pre-to-post-session and the post-session scores were significantly greater for relatives compared with patients (for all comparisons, $P<0.039$ ). Notably, post-session scores of patients were reliably (positively) associated with those of their relatives (where a patient had two relatives, the mean score was calculated) in all domains: FND understanding $r(102)=0.27, P=0.006$; agreement with diagnosis $r(100)$ $=0.23, P=0.021$; hopefulness of recovery $r(99)=0.31, P=0.001$; belief in FND treatability $r(100)=0.31, P=0.002$. Degree of pre- to post-session change (residualised changes scores) was not, however (for all correlations, $r>-0.08$ and $<0.18, P>0.091$ ).

\section{[Insert Table 3 about here]}

Ratings obtained on feedback forms indicated high satisfaction with the session (Figure 2), with mean scores greater than 4 (out of 5 ) for all measures except FND patients for perceived helpfulness. Notably, relative ratings were significantly higher than FND patients for perceived helpfulness and recommendation, with differences on overall satisfaction approaching significance $(P=0.068)$.

[Insert Figure 1 about here] 


\section{Associations with pre-to-post session changes on outcome measures in patients}

and relatives

The mean patient change scores from pre-to-post session on outcome measures, according to whether or not patients attended the session with a relative, are shown in Figure 3. There were no significant differences in change scores (RCS) on any measure, although there was a trend $(P=0.060)$ for patients attending with one or two relatives to show greater change concerning agreement with diagnosis compared to those patients attending without a relative. Additionally, aside from a modest inverse correlation between FND duration and RCS on FND understanding, $r(158)=-0.158, P=0.047$, patients' degree of change on each measure was unrelated to age, gender, education, duration of illness, self-reported symptomatology (e.g. presence of fits or seizures, muscle spasms, tingling or numbness etc.) and EQ Health score (for all associations $P$ > 0.09).

[Insert Figure 2 about here]

Interestingly, younger relatives (18-35 years: $M=23.04, S D=27.54$ ) were more likely to show greater change concerning agreement with diagnosis than older relatives (36-55 years: $M=14.19, \mathrm{SD}=24.30 ; 56+$ years: $M=19.12, \mathrm{SD}=22.75$; comparison of RCS: $P$ $=0.025)$. But there were no significant relationships observed between magnitude of change on outcome measures and age, gender or relationship with the patient (i.e., parent, partner, or other relative (including sibling)/friend; for all associations, $P>0.07$ ).

\section{Discussion}


Following a multi-professional and expert-patient delivered education session for people diagnosed with FND, we found significant increases in ratings of understanding of diagnosis, belief in treatability, hopefulness regarding recovery, and agreement with diagnosis, for both patients and their relatives. The largest effect size was found for increased understanding of the diagnosis. Of note, post-session scores of patients were positively associated with those of their relatives, and there was a trend for patients who attended with relative(s) to report greater change in terms of agreement with diagnosis compared to those who attended without a relative. This suggests that increasing knowledge of FND within patients' wider context is beneficial. Mean satisfaction data was high, with mean ratings of greater than 4 on all measures, with the exception of mean helpfulness ratings given by patients (mean=3.1). Relative ratings were significantly higher than patients in terms of helpfulness ratings and whether they would recommend the session. This may be due to relatives having limited prior knowledge of FND.

The mean number of patients in each education session was 18, illustrating that the group format was an efficient way of giving enhanced information, in terms of both time and access to different professionals. Although cost-effectiveness was not formally evaluated in this study, it is possible that an intervention of this kind can bring costsavings. Most patients brought a relative with them to the session (68\%) suggesting that this was a well-valued option. In line with expected demographics of FND patients, more patients were female (79\%) than male [1]. EQ-5D-5L scores were impaired compared to age-matched healthy UK populations, indicating high distress and disability associated with diagnosis. Most participants self-reported more than one neurological symptom $(80 \%)$, and nearly half of all participants reported 4 or more symptoms $(44 \%)$. This is consistent with a recent study of 322 patients with functional motor disorder, where most patients had more than one functional symptom [25].

It is well established that communication of diagnosis is an important part of the treatment pathway in FND $[6,8]$. Good communication can result in decreased symptoms and 
reduced healthcare use $[6,9,10]$; and theoretically could also result in increased engagement with future treatment, and reduced stigma and distress associated with diagnosis. This can be quite time intensive process if delivered individually. Results from our evaluation indicate that hopefulness and belief in treatability were increased, suggesting possible reduced distress and stigma associated with condition, as well as possible improved readiness for treatment. Longitudinal studies are required in order to establish whether enhancing knowledge of FND in this way results in greater engagement with treatment, reduced symptoms, and/or reduced stigma and distress associated with diagnosis. Limitations of our study include a lack of control group, no test of prior FND knowledge, and no evaluation of possible intervention effects over time (e.g. impact on symptoms, engagement in treatment). It is also unclear how long the increase in knowledge and understanding lasted for. A future study could repeat the outcome measures at a later time point, for example, one month post-session. Additionally, although participants were given questionnaires during the question and answer session, so would have unlikely conferred with one another, it is possible patients and their relatives discussed their answers with one another, potentially influencing responses. Nevertheless, our results demonstrate that a multi-disciplinary single group education session is an effective and acceptable method of increasing understanding and acceptance of a FND diagnosis. In our view, this can aid readiness for future management and treatment.

Funding: None

Data sharing: The data that support the findings of this study are available from the corresponding author upon reasonable request.

Acknowledgments: Thank you to the "expert patient" who contributed to the delivery of the education session. 


\section{References}

1 Stone J, Carson A, Duncan R, et al. Who is referred to neurology clinics?-The diagnoses made in 3781 new patients. Clin Neurol Neurosurg 2010;112:747-51. doi:10.1016/j.clineuro.2010.05.011

2 Carson A, Stone J, Hibberd C, et al. Disability, distress and unemployment in neurology outpatients with symptoms 'unexplained by organic disease'. J Neurol Neurosurg Psychiatry 2011;82:810-3. doi:10.1136/jnnp.2010.220640

Shen W, Bowman ES, Markand ON. Presenting the diagnosis of pseudoseizure. Neurology 1990;40:756-756. doi:10.1212/WNL.40.5.756

4 Mellers JDC. The approach to patients with 'non-epileptic seizures'. Postgrad Med J 2005;81:498-504. doi:10.1136/pgmj.2004.029785

5 Duncan R. Psychogenic nonepileptic seizures: diagnosis and initial management. Expert Rev Neurother 2010;10:1803-9. doi:10.1586/ern.10.171

6 Hall-Patch L, Brown R, House A, et al. Acceptability and effectiveness of a strategy for the communication of the diagnosis of psychogenic nonepileptic seizures. Epilepsia 2010;51:70-8. doi:10.1111/j.1528-1167.2009.02099.x

7 Carson A, Lehn A, Ludwig L, et al. Explaining functional disorders in the neurology clinic: a photo story. Pract Neurol 2016;16:56 LP - 61. doi:10.1136/practneurol2015-001242

8 Stone J. Functional neurological disorders: the neurological assessment as treatment. Pract Neurol 2016;16:7 LP - 17. doi:10.1136/practneurol-2015-001241

9 McKenzie P, Oto M, Russell A, et al. Early outcomes and predictors in 260 patients with psychogenic nonepileptic attacks. Neurology 2010;74:64 LP - 69. doi:10.1212/WNL.0b013e3181c7da6a

10 Razvi S, Mulhern S, Duncan R. Newly diagnosed psychogenic nonepileptic seizures: Health care demand prior to and following diagnosis at a first seizure clinic. Epilepsy Behav 2012;23:7-9. doi:10.1016/j.yebeh.2011.10.009 Mayor R, Brown RJ, Cock H, et al. A feasibility study of a brief psycho-educational intervention for psychogenic nonepileptic seizures. Seizure Eur J Epilepsy 
2013;22:760-5. doi:10.1016/j.seizure.2013.06.008

12 Conwill M, Oakley L, Evans K, et al. CBT-based group therapy intervention for nonepileptic attacks and other functional neurological symptoms: A pilot study. Epilepsy Behav 2014;34:68-72. doi:10.1016/j.yebeh.2014.03.012

13 Chen DK, Maheshwari A, Franks R, et al. Brief group psychoeducation for psychogenic nonepileptic seizures: A neurologist-initiated program in an epilepsy center. Epilepsia 2014;55:156-66. doi:10.1111/epi.12481

14 Cope SR, Smith JG, King T, et al. Evaluation of a pilot innovative cognitivebehavioral therapy-based psychoeducation group treatment for functional nonepileptic attacks. Epilepsy Behav 2017;70:238-44.

doi:10.1016/j.yebeh.2017.02.014

15 Whitehead K, Stone J, Norman P, et al. Differences in relatives' and patients' illness perceptions in functional neurological symptom disorders compared with neurological diseases. Epilepsy Behav 2015;42:159-64.

doi:10.1016/j.yebeh.2014.10.031

16 Herdman M, Gudex C, Lloyd A, et al. Development and preliminary testing of the new five-level version of EQ-5D (EQ-5D-5L). Qual Life Res 2011;20:1727-36. doi:10.1007/s11136-011-9903-x

17 Devlin NJ, Shah KK, Feng Y, et al. Valuing health-related quality of life: An EQ-5D5L value set for England. Health Econ 2018;27:7-22. doi:10.1002/hec.3564

18 Cohen J. Statistical analysis for the behavioral sciences. Hillside: : Lawrence Erlbaum 1988.

19 Hair JF, Anderson RE, Tatham RL, et al. Multivariate Data Analysis: International Edition. 5th ed. Prentice Hall 1998.

20 Efron B, Tibshirani RJ. An introduction to the bootstrap. New York: : Chapman \& Hall 1993.

21 Sakia R. The Box-Cox transformation technique: a review. J R Stat Soc Ser D (The Stat 1992;41:169-78.

22 Benjamini $\mathrm{Y}$, Hochberg $\mathrm{Y}$. Controlling the false discovery rate: a practical and 
powerful approach to multiple testing. J R Stat Soc Ser B 1995;57:289-300.

23 Kind P, Hardman G, Macran S. UK population norms for EQ-5D. Cent Heal Econ Univ York, Work Pap 1999.

24 Marten O, Brendan M, Bansback N, et al. Implausible states: prevalence of EQ-5D$5 \mathrm{~L}$ states in the general population and its effect on health state valuation. Med Decis Mak 2020.

25 O'Connell N, Nicholson TR, Wessely S, et al. Characteristics of patients with motor functional neurological disorder in a large UK mental health service: A case-control study. Psychol Med 2020;50:446-55. doi:10.1017/S0033291719000266

Figure Captions

Figure 1. Session satisfaction ( $0=$ 'Not at all satisfied', $5=$ 'Very satisfied'), perceived helpfulness ( $0=$ 'Not at all', $5=$ 'Very much') and recommendation ( $0=$ 'Unlikely', $5=$ 'Very likely') for FND patients and relatives attending the session. Note: Numbers of patients and relatives providing response were slightly variable for each measure; Data labels represent mean scores; Error bars represent 95\% confidence intervals; Asterisks indicate significant differences between patient and relative groups, ${ }^{*} P<0.05$, ${ }^{* * *} P<$ 0.001 .

Figure 2. Mean change scores from pre-to-post session on study measures in patients attending with and without relatives; Data labels represent mean change scores; Error bars represent $95 \%$ confidence intervals. 


\section{Table 1}

Socio-demographic data of FND patients $(N=193)$ and relatives $(N=152)$ participants attending the session.

\begin{tabular}{lll}
\hline Variable & FND patients & Relatives \\
Female / Male & $152(78.8) / 41(21.2)$ & $71(53.0) / 63(47.0)$ \\
Age (years) & & \\
$18-25$ & $32(16.7)$ & $11(8.5)$ \\
$26-35$ & $40(20.8)$ & $16(12.3)$ \\
$36-45$ & $42(21.9)$ & $21(16.2)$ \\
$46-55$ & $46(24.0)$ & $34(26.2)$ \\
$56-65$ & $22(11.5)$ & $26(20.0)$ \\
$66+$ & $10(5.2$ & $22(16.9)$ \\
Ethnicity & & $113(79.6)$ \\
White & $148(77.9)$ & $9(6.3)$ \\
Black & $14(7.4)$ & $13(9.2)$ \\
Asian & $14(7.4)$ & $7(4.9)$ \\
Mixed/Other & $14(7.4)$ & \\
Education level & & $6(5.0)$ \\
Primary School & $7(4.1)$ & $39(32.2)$ \\
GCSE or Equivalent & $54(31.4)$ & $36(29.8)$ \\
A-Level or Equivalent & $42(24.4)$ & $29(24.0)$ \\
Undergraduate Degree & $47(27.3)$ & $11(9.1)$ \\
Postgraduate Degree & $22(12.8)$ & \\
\hline
\end{tabular}

Please note: Values represent frequency (percentage) unless otherwise stated. Gender information was not available for 18 relatives; Age data was not available for 1 patient and 22 relatives;

Education data was not available for 21 patients and 31 relatives; Ethnicity data was not available for 3 patients and 10 relatives; Data on FND duration and symptoms was not available for 6 and 3 patients, respectively; Percentages were calculated from samples that included only patients/relatives for which data was available. 


\section{Table 2}

Clinical profile of FND $(N=193)$ participants.

FND duration (months)

Self-reported symptomatology

Fits or seizures

Muscle spasms

Tingling or numbness

Limb weakness

Difficulty walking

Memory problems

Other

Two or more symptoms

Four or more symptoms

Six or more symptoms

EQ-5D-5L

EQ Health $(-0.285-1.00)$

$\%$ reporting problems

Mobility

Self-care

Usual activities

Pain/Discomfort

Anxiety/Depression
$\mathrm{Med}=48.0(\mathrm{IQR}=18.0-96.0 ;$ range $1-480)$

n $(\%)$

83 (43.7)

$100(52.6)$

98 (51.6)

104 (53.9)

87 (45.8)

$104(54.7)$

58 (30.5)

$154(81.1)$

84 (44.2)

24 (12.6)

Mean (SD)

$0.553(0.297)$

n (\%)

107 (61.1)

$72(41.1)$

$146(83.4)$

$133(75.6)$

$139(80.3)$

Please note: Values represent frequency (percentage) unless otherwise stated. Med = median; IQR $=$ interquartile range; $\mathrm{SD}=$ standard deviation; EQ Health $=\mathrm{EQ}-5 \mathrm{D}-5 \mathrm{~L}$ health state evaluation; EQ5D-5L data was available for 173 patients; FND symptomatology was not reported by 3 participants; Percentages were calculated from samples that included only patients for which data was available. 


\section{Table 3}

Mean (SD) scores and (within group) effect size across measures of understanding and beliefs about FND in patients and relatives pre- and post-education session.

\begin{tabular}{|c|c|c|c|c|c|}
\hline & Pre-session & Post-session & \multicolumn{3}{|c|}{ Pre- versus Post-session } \\
\hline & Mean (SD) & Mean (SD) & Mean difference $(95 \% \mathrm{CI})$ & $P$ & $d$ \\
\hline \multicolumn{6}{|c|}{ Understand FND (0-100) } \\
\hline Patients $(N=161)$ & $45.81(27.15)$ & $72.92(20.93)$ & $27.11(23.02,31.20)$ & $<0.001$ & 1.00 \\
\hline Relatives $(N=115)$ & $40.14(26.38)$ & $77.39(16.15)$ & $37.26(32.83,41.54)$ & $<0.001$ & 1.52 \\
\hline \multicolumn{6}{|c|}{ Agree with diagnosis (0-100) } \\
\hline Patients $(N=156)$ & $63.65(28.96)$ & $73.56(26.54)$ & $9.90(6.35,13.46)$ & $<0.001$ & 0.33 \\
\hline Relatives $(N=109)$ & $64.41(29.13)$ & $83.58(18.18)$ & $19.17(14.53,24.24)$ & $<0.001$ & 0.69 \\
\hline \multicolumn{6}{|c|}{ Hopeful of recovery $(0-100)$} \\
\hline Patients $(N=162)$ & $60.47(30.68)$ & $65.62(29.50)$ & $5.15(2.38,7.92)$ & $<0.001$ & 0.17 \\
\hline Relatives $(N=109)$ & $70.51(27.17)$ & $80.46(21.46)$ & $9.95(5.88,14.09)$ & $<0.001$ & 0.37 \\
\hline \multicolumn{6}{|c|}{ Believe FND treatable (0-100) } \\
\hline Patients $(N=158)$ & $56.01(27.55)$ & $71.04(25.21)$ & $15.03(11.61,18.45)$ & $<0.001$ & 0.55 \\
\hline
\end{tabular}

This article is protected by copyright. All rights reserved 
$\mathrm{CI}=$ confidence interval; $d=$ Cohen's $d$ : small effect size $=0.2 ;$ medium $=0.5 ;$ large $=0.8$ (Cohen, 1988).

This article is protected by copyright. All rights reserved 


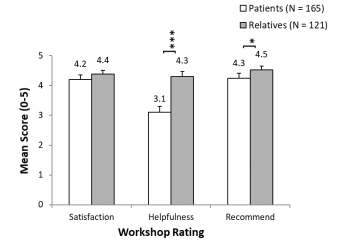

ene_14525_f1.tif

This article is protected by copyright. All rights reserved 


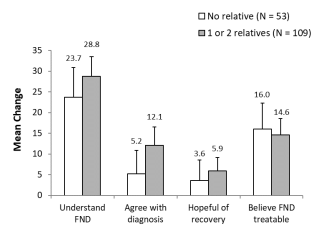

ene_14525_f2.tif

This article is protected by copyright. All rights reserved 